\title{
1 Development of Natto with germination-defective mutants of 2 Bacillus subtilis (natto)
}

3

4 Nobuo Mitsui · Hisashi Murasawa · Junichi Sekiguchi

5

6

7

8

9

10

$11 \quad$ N. Mitsui $\cdot$ J. Sekiguchi $(\bowtie)$

12 Department of Bioscience and Textile Technology, Interdisciplinary Graduate School of 13 Science and Technology, Shinshu University, 3-15-1 Tokida, Ueda-shi, Nagano 14 386-8567, Japan

15 e-mail: jsekigu@shinshu-u.ac.jp

16

17

18 N. Mitsui $\cdot$ H. Murasawa

19 Food Research Laboratory, Asahimatsu Foods Co., Ltd., 1008 Dashina, Iida-shi, 20 Nagano 399-2561, Japan 21 
1 Abstract The effects of cortex-lysis related genes with the pdaA, sleB 2 and $c w l D$ mutations of Bacillus subtilis (natto) NAFM5 on sporulation and 3 germination were investigated. Single or double mutations did not prevent 4 normal sporulation, but did affect germination. Germination was severely 5 inhibited by the double mutation of $s l e B$ and $c w l D$. The quality of natto 6 made with the sleB $c w l D$ double mutant was tested, and the amounts of 7 glutamic acid and ammonia were very similar to those in the wild type. The 8 possibility of industrial development of natto containing a reduced number 9 of viable spores is presented.

10

11 Keywords Germination $\cdot \operatorname{sleB} \cdot c w l D \cdot p d a A \cdot B a c i l l u s$ subtilis $\cdot$ natto 


\section{Introduction}

2

3 Natto is a traditional and popular food in Japan (Murooka and Yamashita 2008). It has unique features regarding stickiness and taste, which are related to the digestion of soy proteins. It is made from steamed soybeans using Bacillus subtilis (natto) as a starter. $B$. subtilis (natto) forms spores that are found in the natto. Bacillus spores are extremely stable as to chemical and/or physical treatments. Therefore, the killing of spores in natto is not easy without loss of food quality, i.e. taste, flavor, color and/or stickiness. For these reasons, the utilization of natto for processed foods has not increased, and natto containing a decreased amount of spores has been desired. For the industrial development of natto, sporulation and/or germination mutants are needed as starters. Since enzymes produced during the sporulation phase usually affect the quality of natto, germination mutants are expected to be the strongest candidates. In B. subtilis, various germination-deficient mutants have been investigated. The major group of germination-deficient mutants is related to the germination receptors for germinants [L-alanine, AGFK (a mixture of L-asparagine, D-glucose, D-fructose and $\mathrm{KCl}$ ), and $\mathrm{Ca}^{2+}$-dipicolinic acid]. This group contains gerA, ger B, gerK, and gerD (Paidhungat and Setlow 2002; Ragkousi et al. 2003). The mutants of this group are inadequate for our purpose, because germinants comprise not only L-alanine, AGFK, and $\mathrm{Ca}^{2+}$-dipicolinic acid but also possible unknown nutrients (inosine is known as a good germinant for other bacilli; Moir 1992). The second group is related to spore coat deficiency. The gerT and ger $Q$ mutants belong to this group but their spores germinate at high frequency on rich media (Ragkousi et al. 2003; Ferguson et al. 2007). The third group affects vegetative growth and/or sporulation. The ger $C$, ger $F$, ger $G$, and gerE mutants belong to this group (Paidhungat and Setlow 2002; Piggot and Losick 2002; Moir 1981). These mutants are also inadequate, because the mutants are not clear germination-defective mutants and may affect the quality of natto. The fourth group is related to cortex-lysis or modification, and it contains $c w l D$, sleB, cwlJ, and pdaA (Sekiguchi et al. 1995; Moriyama et al. 1996; Ishikawa et al. 1998). CwlD, SleB, and CwlJ were considered to be cortex-lytic enzymes. SleB recognizes muramic-delta-lactam, which is a unique peptidoglycan component in the cortex (Warth and Strominger 1969). Muramic-delta-lactam is produced by PdaA (polysaccharide deacetylase) and CwID ( $N$-acetylmuramoyl-L-alanine amidase homologue) (Sekiguchi et al. 1995; Fukushima et al. 2002; Gilmore et al. 2004; Fukushima et al. 2005). The mutations in the fourth group affect neither vegetative growth nor sporulation, but severely affect germination. Therefore, in this study we investigated the effect of disruption of $p d a A, c w l D$ and/or 
$1 \quad$ sleB on the germination of B. subtilis (natto), and applied the mutants to the production 2 of natto. 


\section{Materials and methods}

2

3 Bacterial strains and plasmids

The strains of B. subtilis 168, B. subtilis (natto) NAFM5 (Kimura et al. 2004), and Escherichia coli, and the plasmids used in this study are listed in Table 1. NAFM5 was a derivative of the major natto starter, Miyagino strain, and NAFM5 had the competence ability. B. subtilis was grown on LB medium (Sambrook et al. 1989) at $37^{\circ} \mathrm{C}$ overnight, inoculated into DSM (Schaeffer sporulation medium) (Schaeffer et al. 1965), and then shaken at $37^{\circ} \mathrm{C}$. If necessary, kanamycin and tetracycline were added to the medium to final concentrations of 20 and $10 \mu \mathrm{g} \mathrm{ml}^{-1}$, respectively. E. coli was grown in LB medium at $37^{\circ} \mathrm{C}$. If necessary, kanamycin, tetracycline, and ampicillin were added to the medium to final concentrations of 20,20 , and $50 \mu \mathrm{g} \mathrm{ml}^{-1}$, respectively.

\section{Plasmids construction}

To construct $p d a A$ mutants, the upstream region of $p d a A$ was amplified by PCR using two primers, pdaAUP1 and pdaAUP2 (Table 2), with B. subtilis (natto) NAFM5 DNA as a template. The PCR fragment was digested with $K p n \mathrm{I}$ and BamHI, and ligated to pUC118 at the corresponding sites, followed by transformation of E. coli JM109, plasmid pDA1 being generated. pDG780 was digested with BamHI and SalI, and the $1.5 \mathrm{~kb}$ fragment (kanamycin cassette) was inserted into pDA1 at the corresponding sites to generate plasmid pDA2. Similarly, the downstream region of $p d a A$ was cloned into pUC118 with two primers, pdaADW1 and pdaADW2, to generate plasmid pDA3. pDA2 was digested with KpnI and Sall, and the $2.5 \mathrm{kbp}$ fragment was inserted into pDA3 at the corresponding sites. The resulting plasmid, pDA4, was used to construct a pdaA-deficient mutant. pDA4-Tc containing the tetracycline cassette from pDG1515 instead of the kanamycin cassette of pDA4 was used to construct a pdaA sleB-double mutant.

Likewise, for sleB mutants, pSB4 was constructed using the upstream and downstream regions of $s l e B$ and the kanamycin cassette from pDG780. The fragment was amplified by PCR with $B$. subtilis (natto) NAFM5 DNA as a template and primers sleBUP1 and sleBUP2, or primers sleBDW1 and sleBDW2. For $c w l D$ mutants, pCD4 was constructed using the upstream and downstream regions of $c w l D$ and the tetracycline cassette from pDG1515. The fragment was amplified by PCR with $B$. subtilis (natto) NAFM5 DNA as a template and primers cwlDUP1 and cwlDUP2, or 
1 primers cwlDDW1 and cwlDDW2.

2

3 Mutant construction

4

5 pdaA-deficient mutants, $16 \mathrm{~A}$ and NAA, were constructed by transformation of $B$.

6 subtilis 168 and B. subtilis (natto) NAFM5, respectively, with pDA4. Disruption of the

$7 \quad p d a A$ gene was confirmed by PCR using primers pdaAUP1 and pdaADW2.

8 sleB-deficient mutants, 16B and NAB, were constructed by transformation of 168 and

9 NAFM5, respectively, with pSB4. cwlD-deficient mutants, 16D and NAD, were

10 constructed by transformation of 168 and NAFM5, respectively, with pCD4. pdaA and

11 sleB double-deficient mutants, $16 \mathrm{AB}$ and $\mathrm{NAAB}$, were constructed by transformation of

$1216 \mathrm{~B}$ and $\mathrm{NAB}$, respectively, with pDA4-Tc. pdaA cwlD-double mutants, 16AD and

13 NAAD, were constructed by transformation of 16A and NAA, respectively, with pCD4.

14 sleB $c w l D$-double mutants, 16BD and NABD, were constructed by transformation of

$1516 \mathrm{~B}$ and NAB, respectively, with pCD4.

16

17 Transformation of E. coli and B. subtilis strains

19 E. coli transformation was performed as described by Sambrook et al. (1989), and $B$.

20 subtilis and B. subtilis (natto) transformation was performed by the procedure of

21 Anagnostopoulos and Spizizen (1961).

Viable spore counts

B. subtilis 168, 16A, 16B, and 16D, and B. subtilis (natto) NAFM5, NAA, NAB, and

26 NAD were shake-cultured in LB medium overnight at $37^{\circ} \mathrm{C}$. These precultures were 27 inoculated into DSM followed by shake-culturing for 3 days at $37{ }^{\circ} \mathrm{C}$. The numbers of spores were determined with a bacterial counting chamber on a microscope. Heat resistance spores $\left(80^{\circ} \mathrm{C}, 10 \mathrm{~min}\right)$ were counted by a standard agar method (Nissui Pharm. Co.) as viable colonies after 3 days at $37^{\circ} \mathrm{C}$. 
Spore germination

3 B. subtilis 168, 16AB, 16AD, and 16BD, and B. subtilis (natto) NAFM5, NAAB,

4 NAAD, and NABD were shake-cultured in DSM for 3 days at $37{ }^{\circ} \mathrm{C}$. The spores were

5 purified by lysozyme treatment followed by salt and detergent washes as described by

6 Nicholson and Setlow (1990). The purified spores were adjusted to $A_{600}=1$ and their

7 viability was assayed described above. To measure spore germination as the loss of

8 optical density and release of dipicolinic acid, spores were diluted with a $10 \mathrm{mM}$

9 Tris-HCl buffer ( $\mathrm{pH}$ 8.4). Germination was initiated by the addition of L-Ala (10 mM).

10 At appropriate times, $\mathrm{A}_{600}$ of the mixture was measured, and a $3 \mathrm{ml}$ sample was taken

11 and centrifuged with a microcentrifuge. The supernatant was used for the measurement

12 of released dipicolinic acid as described by Nicholson and Setlow (1990).

14 Analysis of natto

16 B. subtilis (natto) NAFM5 and NABD were shake-cultured in LB medium at $37{ }^{\circ} \mathrm{C}$ 17 overnight, and then the cultures were diluted to $10^{5} \mathrm{cells} \mathrm{ml}^{-1}$ with saline. To make natto, $181 \mathrm{ml}$ of a diluent was inoculated into $50 \mathrm{~g}$ of autoclaved soybeans $\left(121^{\circ} \mathrm{C}, 50 \mathrm{~min}\right)$, 19 followed by fermentation at $40^{\circ} \mathrm{C}$ for $17 \mathrm{~h}$. One hundred $\mathrm{ml}$ of saline were added to the 20 natto, followed by agitating for $1 \mathrm{~h}$ at $4{ }^{\circ} \mathrm{C}$. Then the supernatant was used to count the 21 viable spores, and to measure the glutamate and ammonia contents for evaluation of the 22 natto. The amounts of glutamate and ammonia were determined with an F-kit 23 L-glutamic acid (Roche) and an F-kit ammonia, respectively, according to the 24 manufacturer's instructions. The organoleptic evaluation of stickiness and flavor were 25 performed by lifting the natto after mixing with a spatula. 


\section{Results}

2

3 Effects of single mutants as to cortex-lysis related genes

5 SleB is a cortex-lytic enzyme (Moriyama et al. 1996) and is assumed to digest the

Effects of double mutants as to cortex-lysis related genes

Although the single mutants as to the cortex-lysis related genes showed decreased numbers of viable spores, the effects were not complete. Therefore, we examined the effect of double mutants as to cortex-lysis related genes, i.e. the $p d a A$ and sleB, $p d a A$ 
1 and $c w l D$, and $s l e B$ and $c w l D$ mutations. All the double mutants formed spores at 2 frequencies similar to in the single mutants. To analyze the germination of mutants, the 3 spores were purified and then they $\left(\mathrm{A}_{600}=1\right)$ were heated for $10 \mathrm{~min}$ at $80^{\circ} \mathrm{C}$, followed 4 by plating on agar medium to determine viable spore numbers (Fig. 2). Colonies were counted after 3 days, because the colonies of mutants were very small at $24 \mathrm{~h}$.

The most dramatic effect was found for the double mutant as to sleB and $c w l D . B$. subtilis 168 spores with these mutations gave $8.6 \times 10^{2}$ viable cells $\mathrm{ml}^{-1}$, and $B$. subtilis (natto) spores gave $1.0 \times 10^{2}$ viable cells $\mathrm{ml}^{-1}$ (Fig. 2B). Since the sleB gene product was considered to only degrade the cortex with muramic-delta-lactam (Masayama et al. 2006), the effects of the mutations were different from each other (sleB is not associated with muramic-delta lactam formation). Therefore, the synergetic effect of the sleB and cwlD mutations was found for both B. subtilis 168 and B. subtilis (natto). From a practical aspect, the $s l e B c w l D$-double mutant is a good candidate for producing natto with spores deficient in significant germination.

Analysis of spore germination

Since the $s l e B c w l D$-double mutants exhibited the lowest germination, germination was compared among 168, 16BD ( $\Delta$ sleB $\Delta c w l D)$, NAFM5, and NABD $(\Delta s l e B \Delta c w l D)$ spores (Fig. 3). During germination in L-Ala buffer, the 16BD and NABD spores showed a slower decrease in $\mathrm{A}_{600}$ than the wild type spores of 168 and NAFM5, respectively. Therefore, the germination of both strains was severely affected by the double mutations. Dipicolinic acid release from spores is also a marker of the early germination event. But the levels of the release of dipicolinic acid from these spores were almost the same as that in the wild type. These results suggest that an early germination event (i.e., dipicolinic acid release) was not affected by the double mutations in either strain. These data were supported by the results for each single mutation in B. subtilis (Sekiguchi et al. 1995; Moriyama et al. 1996). Phase-contrast microscopy of the wild type and mutants is shown in Fig. 4. The refractility of spores of the wild types, 168 and NAFM5, changed from bright to dark after $6 \mathrm{~h}$, whereas 16BD and NABD spores became phase gray (or blight) under the same conditions. After $24 \mathrm{~h}$, the 16BD and NABD spores were still phase gray (Fig. 4F and $\mathrm{H}$ ). These results correspond to the spore viability and the loss of optical density. On the other hand, it is interesting that the dipicolinic acid content of B. subtilis (natto) spores was 1.5 times larger that that of B. subtilis ones (Fig. 3B). 
2 Analysis of natto

3

4 Since the sleB $c w l D$-double mutants formed spores but viable spores were very few, we 5 made natto with NAFM5 and NABD, and then determined the numbers of spores and 6 quality of natto (Fig. 5). Microscopy of extracts of natto indicated that the spore 7 formation of NABD was normal, but the viable spore number decreased similarly in a 8 liquid culture (Fig. 5). The organoleptic test of stickiness and flavor, and both the 9 glutamic acid and ammonia contents of NABD were similar to those of the wild type 10 (Fig. 5 and data not shown). These results indicated that the natto made with NABD 11 exhibited normal qualities. 


\section{Discussion}

2

3

Spores of $B$. subtilis were extremely stable as to chemical or physical attack. Natto is a soybean-fermented food and B. subtilis (natto) is used as a starter, therefore natto contains many spores. On the other hand, low bacterial numbers in processed foods are desirable from the viewpoint of shelf life. For this reason, natto is not used as a processed food. Spores from natto contaminate machinery used for manufacturing foods and thus the foods have high initial viable cell counts. Therefore, natto with no or few spores is required.

There have been many reports about germination-defective mutants of B. subtilis. Fukushima et al. (2002) reported that the germination rate of pdaA mutants was $0.0008 \%$. In this study, the germination rates of the pdaA mutants were $0.075 \%$ and $0.17 \%$ for 168 and NAFM5, respectively. The germination rate of the NAFM5 mutant was apparently higher than that of the 168 mutant. The germination rate of the 168 mutant shown in this study was higher than that previously reported. Ishikawa et al. (1998) reported the germination rate of the sleB mutant in B. subtilis was $43 \%$, and our results were $61 \%$ and $1.2 \%$ for 168 and NAFM5, respectively. The mutation of sleB was more effective in NAFM5 than in 168. Sekiguchi et al. (1995) reported that the germination rate of the $c w l D$ mutant in B. subtilis was less than $3.7 \times 10^{-8}$, but our results were $1.9 \times 10^{-4}$ and $1.7 \times 10^{-3}$ for 168 and NAFM5, respectively. Popham et al. (1996) showed that the germination rate of the cwlD mutant in B. subtilis was $5 \times 10^{-4}$ (with the respect to the wild type).

We investigated the effects of double mutations of these three genes. The $c w l D$ and sleB double mutation strongly affected the germination frequency. The loss of optical density of the spores of the $c w l D$ sleB-double mutant was low compared with in the wild type, and the refractility of the spores stopped at the phase gray stage. But the DPA release level was almost the same as that in the wild type. These results indicate that the germination of the $c w l D$ sleB-double mutant was blocked at a late stage of germination. This phenomenon resembled the case of the $s l e B c w l J$-double mutant (Ishikawa et al. 1998). The release of dipicolinic acid of NAFM5 was higher than that of 168 . The reason was not clear but the spore size was larger than that of 168 (Fig. 4). The difference of DPA release may be attributed to the different spore size.

Stickiness, and the amounts of glutamic acids and ammonia are important for natto qualities. The natto made with the $c w l D$ sleB-double mutant has the same qualities as the wild type. This indicates that the $c w l D$ sleB-double mutant has no effect on natto qualities. But natto contains vegetative cells in addition to spores. The vegetative cells 
1 in natto also affect the shelf life of processed foods. Therefore, process to decrease the 2 number of vegetative cells is required. Such processes are heating, freezing, and 3 addition of preservatives such as glycine or lysozyme, and they are milder than killing 4 spores. Consequently, the effect on food quality seems to be very little. Similarly, 5 decolonization of vegetative cells contaminated on machine is easier than that of spores.

6 The introduction of a cold-sensitive mutation to the germination mutant may become an 7 improvement process. Moreover, natto contains abundant vitamin $\mathrm{K}$ that is expected to 8 prevent osteoporosis. However, because vitamin $\mathrm{K}$ weakens the effect of an anti-blood 9 coagulant pharmaceutical agent, it is not preferable that patients taking the agent have 10 natto (Homma et al. 2006). It is known that B. subtilis spores germinate in intestines 11 (Casula G and Cutting SM 2002). It means B. subtilis produces vitamin $\mathrm{K}$ in intestines 12 (Kaneki et al. 2001). In contrast to spores, vegetative cells do not have the tolerance to 13 peptic juice (Duc et al. 2003). Therefore, the control of the germination of spores in 14 intestines is preferable and thus the germination mutant may be able to be used for its 15 application. In this manuscript, we present the possibility of the improved natto 16 production with a $s l e B c w l D$ germination-defective mutant.

\section{Acknowledgements}

20 The authors would like to thank Dr. Keitarou Kimura (National Food Research Institute) 21 for the supply of B. subtilis (natto) NAFM5. This work was supported by 22 Grants-in-Aid for Scientific Research (B) (19380047) and for the Global COE program 23 of the Ministry of Education, Culture, Sports, Science, and Technology of Japan 24 (MECSSTJ). 


\section{References}

Anagnostopoulos C, Spizizen J (1961) Requirements for transformation in Bacillus subtilis. J Bacteriol 81:741-746

Casula G, Cutting SM (2002) Bacillus probiotics: spore germination in the gastrointestinal tract. Appl Environ Microbiol 68:2344-2352

Duc le H, Hong HA, Cutting SM (2003) Germination of the spore in the gastrointestinal tract provides a novel route for heterologous antigen delivery. Vaccine 21:4215-4224

Ferguson CC, Camp AH, Losick R (2007) gerT, a newly discovered germination gene under the control of the sporulation transcription factor $\sigma^{\mathrm{K}}$ in Bacillus subtilis. $\mathrm{J}$ Bacteriol 189:7681-7689

Fukushima T, Kitajima T, Sekiguchi J (2005) A polysaccharide deacetylase homologue, $\mathrm{PdaA}$, in Bacillus subtilis is $\mathrm{N}$-acetylmuramic acid deacetylase in vitro. J Bacteriol 187:1287-1292

Fukushima T, Yamamoto H, Atrih A, Foster SJ, Sekiguchi J (2002) A polysaccharide deacetylase gene $(p d a A)$ is required for germination and for production of muramic delta-lactam residues in the spore cortex of Bacillus subtilis. J Bacteriol 184:6007-6015

Gilmore ME, Bandyopadhyay D, Dean AM, Linnstaedt SD, Popham DL (2004) Production of muramic delta-lactam in Bacillus subtilis spore peptidoglycan. J Bacteriol 186:80-89

Homma K, Wakana N, Suzuki Y, Nukui M, Daimatsu T, Tanaka E, Tanaka K, Koga Y, Nakajima Y, Nakazawa H (2006) Treatment of natto, a fermented soybean preparation, to prevent excessive plasma vitamin $\mathrm{K}$ concentrations in patients taking warfarin. J Nutr Sci Vitaminol (Tokyo) 52:297-301

Ishikawa S, Yamane K, Sekiguchi J (1998) Regulation and characterization of a newly deduced cell wall hydrolase gene ( $c w l J)$ which affects germination of Bacillus subtilis spores. J Bacteriol 180:1375-1380

Kaneki M, Hodges SJ, Hosoi T, Fujiwara S, Lyons A, Crean SJ, Ishida N, Nakagawa M, Takechi M, Sano Y, Mizuno Y, Hoshino S, Miyao M, Inoue S, Horiki K, Shiraki M, Ouchi Y, Orimo H (2001) Japanese fermented soybean food as the major determinant of the large geographic difference in circulating levels of vitamin K2: possible implications for hip-fracture risk. Nutrition 17:315-321

Kimura K, Tran LS, Uchida I, Itoh Y (2004) Characterization of Bacillus subtilis gamma-glutamyltransferase and its involvement in the degradation of capsule 
poly-gamma-glutamate. Microbiology 150:4115-4123

Masayama A, Fukuoka H, Kato S, Yoshimura T, Moriyama M, Moriyama R (2006) Subcellular localization of a germination-specific cortex-lytic enzyme, SleB, of bacilli during sporulation. Genes Genetic Syst 81:163-169

Moir A (1981) Germination properties of a spore coat-defective mutant of Bacillus subtilis. J Bacteriol 146:1106-1116

Moir A (1992) Spore germination. In: Doi RH, McGloughlin M (eds) Biology of

Moriyama R, Hattori A, Miyata S, Kudoh S, Makino S (1996) A gene (sleB) encoding a

\section{L-alanine-mediated germination. J Bacteriol 178:6059-6063}

Murooka Y, Yamashita M (2008) Traditional healthful fermented products of Japan. J Ind Microbiol Biotechnol 35:791-798

Nicholson, W. L., and P. Setlow. (1990) Sporulation, germination and outgrowth. In: Harwood CR, Cutting SM (eds) Molecular Biological Methods for Bacillus, John Wiley \& Sons, Ltd., Chichester, United Kingdom, pp 391-450

Paidhungat M, Setlow P (2002) Spore germination and outgrowth. In: Sonenshein AL, Hoch JA, and Losick R (eds) Bacillus subtilis and its Closest Relatives: from Genes to Cells, ASM Press, Washington DC, pp 537-548.

Piggot PJ, Losick R (2002) Sporulation genes and intercompartmental regulation. In: Sonenshein AL, Hoch JA, Losick R (eds) Bacillus subtilis and its Closest Relatives: from Genes to Cells, ASM Press, Washington DC, pp 483-517

Popham DL, Helin J, Costello CE, Setlow P (1996) Muramic lactam in peptidoglycan of Bacillus subtilis spores is required for spore outgrowth but not for spore dehydration or heat resistance. Proc Natl Acad Sci USA 93:15405-15410

Qiu D, Fujita K, Sakuma Y, Tanaka T, Ohashi Y, Oshima H, Tomita M, Itaya M (2004) Comparative analysis of physical maps of four Bacillus subtilis (natto) genomes. Appl Environ Microbiol 70:6247-6256

Ragkousi K, Eichenberger P, van Ooij C, Setlow P (2003) Identification of a new gene essential for germination of Bacillus subtilis spores with $\mathrm{Ca}^{2+}$-dipicolinate. $\mathrm{J}$ Bacteriol 185:2315-2329

Sambrook, J., E. F. Fritsch, and T. Maniatis (1989) Molecular Cloning: A Laboratory Manual, 2nd ed. Cold Spring Harbor Laboratory Press, Cold Spring Harbor, N.Y Schaeffer P, Millet J, Aubert JP (1965) Catabolic repression of bacterial sporulation. Proc Natl Acad Sci USA 54:704-711

Sekiguchi J, Akeo K, Yamamoto H, Khasanov FK, Alonso JC, Kuroda A (1995) 
1 Nucleotide sequence and regulation of a new putative cell wall hydrolase gene, $c w l D$, 2 which affects germination in Bacillus subtilis. J Bacteriol 177:5582-5589

3 Warth AD, Strominger JL (1969) Structure of the peptidoglycan of bacterial spores:

4 occurrence of the lactam of muramic acid. Proc Natl Acad Sci USA 64:528-535

5 
1 Figure legends

3 Fig. 1 The spore numbers of the single mutants and the wild types. The single mutants 4 and the wild types of B. subtilis 168 and B. subtilis (natto) NAFM5 were cultured in 5 DSM for 3 days at $37^{\circ} \mathrm{C}$. The numbers of spores were determined under a microscope 6 (A). Heat resistance spores $\left(80^{\circ} \mathrm{C}, 10 \mathrm{~min}\right)$, as viable spores, were plated, followed by 7 counting after 3 days at $37^{\circ} \mathrm{C}(\mathrm{B})$. Open and slashed boxes indicate B. subtilis 168 and 8 B. subtilis (natto) NAFM5, respectively. c.f.u., colony forming units.

Fig. 2 The spore numbers of the double mutants and the wild types. The double mutants and the wild types of $B$. subtilis 168 and B. subtilis (natto) NAFM5 were cultured in DSM for 3 days at $37^{\circ} \mathrm{C}$. The numbers of spores were determined under a microscope (A). Heat resistance spores $\left(80^{\circ} \mathrm{C}, 10 \mathrm{~min}\right)$ were counted after cultivation 15 for 3 days at $37^{\circ} \mathrm{C}$ (B). Open and slashed boxes indicate 168 and NAFM5, respectively.

Fig. 3 The germination properties of the sleB $c w l D$-double mutants and the wild types. The germination of spores of the $s l e B c w l D$-double mutants and the wild types was monitored at $\mathrm{A}_{600}$ at the indicated times after addition to a germination buffer $(10 \mathrm{mM}$ L-alanine, $10 \mathrm{mM}$ Tris- $\mathrm{HCl}(\mathrm{pH} 8.4))$. Relative absorbance is shown in panel A. The dipicolinic acid (DPA) released into the supernatants of spore suspensions during germination is shown in panel B. Open and closed circles indicate 168 and its sleB cwlD-double mutant (16BD), respectively. Open and closed triangles indicate NAFM5

Fig. 4 Phase-contrast microscopy of spores of the sleB $c w l D$-double mutants derived from 168 and NAFM5. Spores were germinated at $37^{\circ} \mathrm{C}$ for $6 \mathrm{~h}$ (panels A to D) or $24 \mathrm{~h}$ (panels $\mathrm{E}$ to $\mathrm{H}$ ) in the germination buffer. Panels A and $\mathrm{E}$ show 168, and panels $\mathrm{B}$ and $\mathrm{F}$ show the $s l e B c w l D$-double mutant of 168 (16BD). Panels C and G show NAFM5, and panels D and H show the sleB $c w l D$-double mutant of NAFM5 (NABD). Bar, $5 \mu \mathrm{m}$. 
1 Fig. 5 The spore numbers and the amounts of glutamic acid and ammonia in natto. 2 Natto made with the $\operatorname{sleB} c w l D$-double mutant and the wild type was added to saline, 3 followed by agitation for $1 \mathrm{~h}$ at $4^{\circ} \mathrm{C}$. (A) The numbers of spores in the supernatants 4 were determined under a microscope or by plating after heat treatment $\left(80^{\circ} \mathrm{C}, 10 \mathrm{~min}\right)$ 5 followed by cultivation for 3 days at $37{ }^{\circ} \mathrm{C}$. (B) The amounts of L-glutamic acid and 6 ammonia in the supernatants were measured with F-kits to evaluate the quality of the 7 natto. Open and slashed boxes indicate NAFM5 and its sleB cwlD-double mutant 8 (NABD), respectively. 
Table 1 Bacterial strains and plasmids used in this study

\begin{tabular}{|c|c|c|}
\hline Strain or plasmid & Genotype & \\
\hline \\
\hline 168 & & D. Ehrlich \\
\hline $16 \mathrm{~A}$ & & $168 \leftarrow$ pDA4 \\
\hline $16 \mathrm{~B}$ & $\operatorname{trp} C 2 \Delta$ sleB:: $\mathrm{Km}^{\mathrm{r}}$ & $168 \longleftarrow p S B 4$ \\
\hline 16D & $\operatorname{trp} C 2 \triangle c w l D:: T^{r}$ & $168 \leftarrow$ pCD4 \\
\hline $16 \mathrm{AD}$ & $\operatorname{trp} C 2 \Delta p d a A:: \mathrm{Km}^{\mathrm{r}} \Delta c w l D:: T c^{\mathrm{r}}$ & $16 \mathrm{~A} \leftarrow \mathrm{pCD} 4$ \\
\hline 16BD & $\operatorname{trp} C 2 \triangle$ sleB $:: \mathrm{Km}^{\mathrm{r}} \triangle \mathrm{cwl} \mathrm{D}: \mathrm{Tc}^{\mathrm{r}}$ & $16 \mathrm{~B} \longleftarrow \mathrm{pCD} 4$ \\
\hline $16 \mathrm{AB}$ & $\operatorname{trp} C 2 \Delta p d a A:: \operatorname{Tc}^{\mathrm{r}} \Delta s l e B:: \mathrm{Km}^{\mathrm{r}}$ & $16 \mathrm{~B} \longleftarrow \mathrm{pDA} 4-\mathrm{Tc}$ \\
\hline \\
\hline NAFM5 & Rif bio & K. Kimura \\
\hline NAA & Rif bio $\Delta p d a A:: \mathrm{Km}^{\mathrm{r}}$ & NA $\leftarrow \mathrm{pDA} 4$ \\
\hline NAB & & $\mathrm{NA} \longleftarrow \mathrm{pSB} 4$ \\
\hline NAD & Rif bio $\triangle c w l D:: T c^{r}$ & $\mathrm{NA} \leftarrow \mathrm{pCD} 4$ \\
\hline NAAD & Rif bio $\triangle p d a A:: \operatorname{Km}^{\mathrm{r}} \Delta c w l D:: \mathrm{Tc}^{\mathrm{r}}$ & NAA $\leftarrow$ pCD 4 \\
\hline NABD & & $\mathrm{NAB} \leftarrow \mathrm{pCD} 4$ \\
\hline NAAB & Rifi bio $\Delta p d a A:: \operatorname{Tc}^{\mathrm{r}} \Delta$ sle $B:: \mathrm{Km}^{\mathrm{r}}$ & $\mathrm{NAB} \longleftarrow \mathrm{pAD} 4-\mathrm{Tc}$ \\
\hline \multirow{3}{*}{ Escherichia coli } & $\operatorname{rec} A 1 \triangle($ lac-proAB $)$ endAI & \\
\hline & & \\
\hline & & \\
\hline & & \\
\hline
\end{tabular}

Plasmid

pUC118

pDG780

pDG1515

pDAl

pDA2

pDA3

pDA4

pDA4-Tc

pSB4

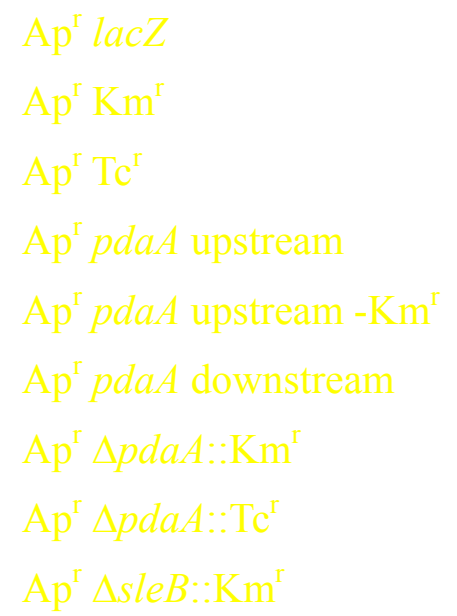

Takara

BGSC

BGSC

This study

This study

This study

This study

This study

This study 
BGSC, Bacillus Genetic Stock Center, Ohio State University

Sources shown before and after the arrows indicate donor plasmid. DNA and recipient cells on transformation, respectively 
Table 2 Primers used in this study

\begin{tabular}{|c|c|c|}
\hline Primer & & Restriction site \\
\hline \multicolumn{3}{|c|}{ pdaA (789bp) } \\
\hline pdaAUP1 & 5'- CGGGGTACC -915 GAAGGTCGGTTTTCTGTCCA_s96 & \\
\hline pdaAUP2 & 5'- CGCGGATCC 29 GCGCA GCATATTGAACACAT $10_{10}$ & BamHI \\
\hline pdaADW1 & 5' - ACGCGTCGAC 772 ATGAGGCTGCCGTCTTTGTA $_{791}$ & \\
\hline & 5' - CCCAAGCTT 1671 GACTCGGCAATATGGGACAG 1652 & HindIII \\
\hline \multicolumn{3}{|c|}{ sleB (915bp) } \\
\hline sleBUP1 & 5'- GCCGAGCTC_-977GAAAACGGCAGGTTTCAC_960 & \\
\hline sleBUP2 & 5’- CGCGGATCC -1 CTTTTCAAGCCTCCTACTGC-20 & BamHI \\
\hline sleBDW 1 & 5'- ACGCGTCGAC 873 GCGTCCGCAGATTAAAAG 890 & \\
\hline sleBDW2 & $5^{\circ}$ - CCCAAGCTT 1870 CTTTAAAAATGCAAGCGCTC 1851 & HindIII \\
\hline \multicolumn{3}{|c|}{ cwlD (711bp) } \\
\hline cwlDUP1 & 5'- GCCGAGCTC -981 CGGGTTTTGTCAATCCTGTT-962 & \\
\hline cw1DUP2 & 5?- CGCGGATCC ${ }_{13}$ GCTTTTTCCTCATCCCTTCC $_{-7}$ & BamHI \\
\hline & 5' - ACGCGTCGAC 695AAGGAGACCCTCCGGAGTAA 714 & \\
\hline & 5'- CCCAAGCTT 1663 TCCCCTGTCTTTGCACTTTC 1644 & HindIII \\
\hline
\end{tabular}

Subscript numbers in the sequence are with respect to the 5'-terminal nucleic acid residues of PdaA, SleB and Cw1D, respectively

Restriction site is underlined 

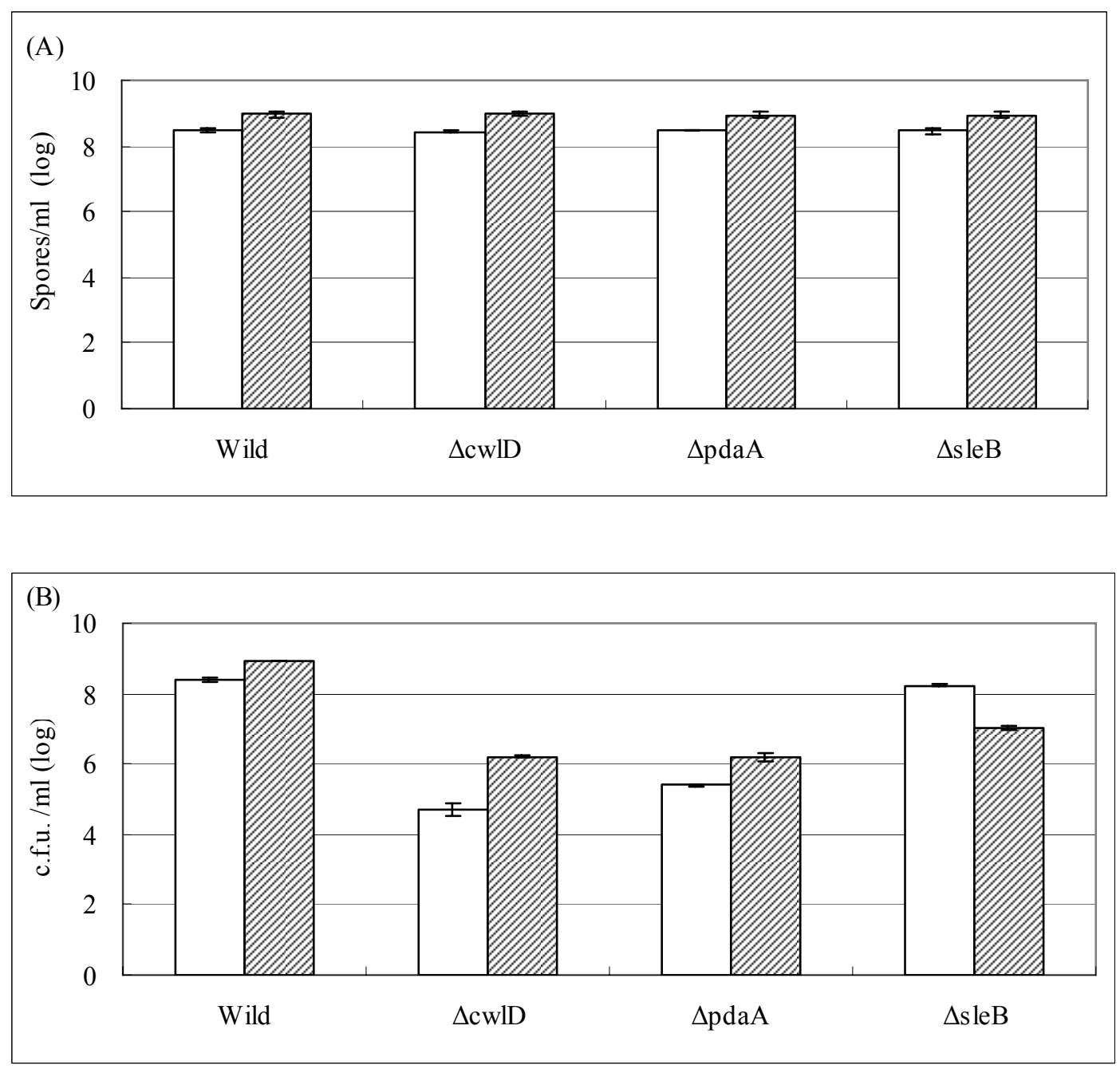

Fig. 1 

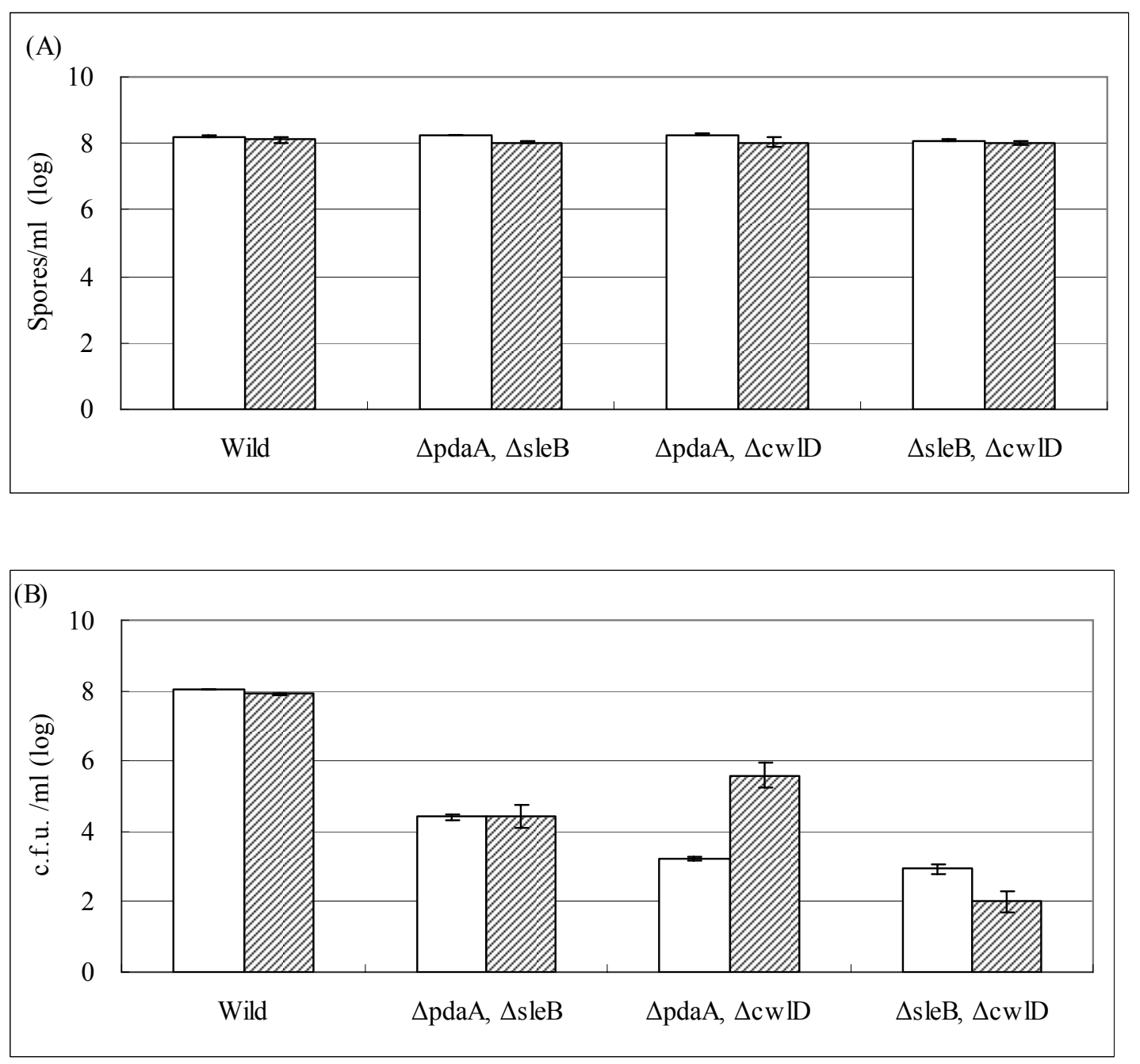

Fig. 2 


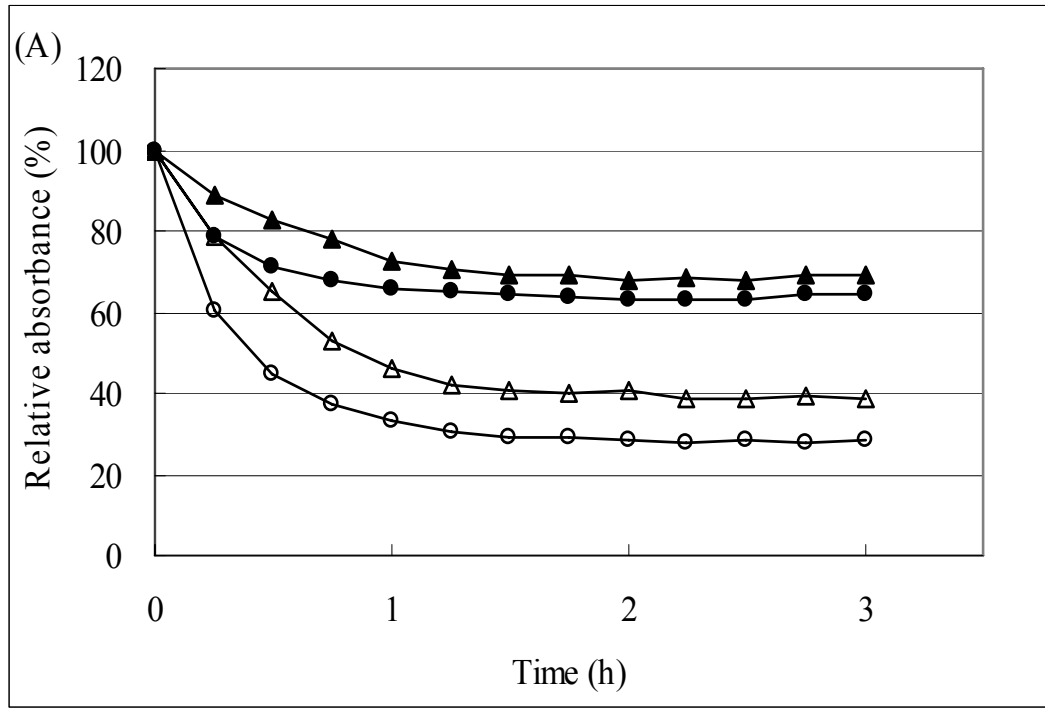

(B)

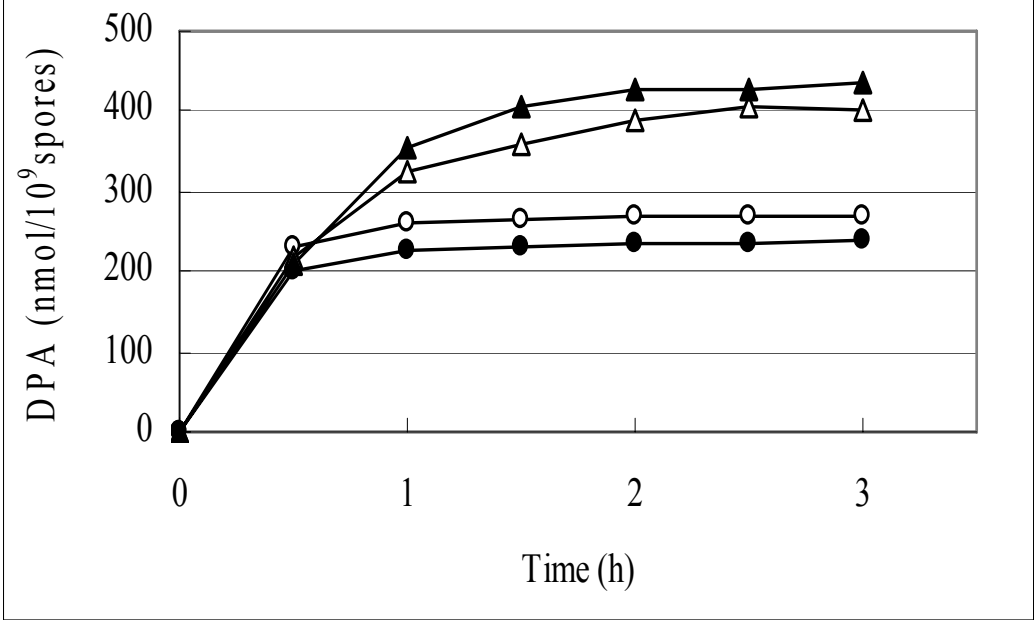

Fig. 3 
$6 \mathrm{~h}$
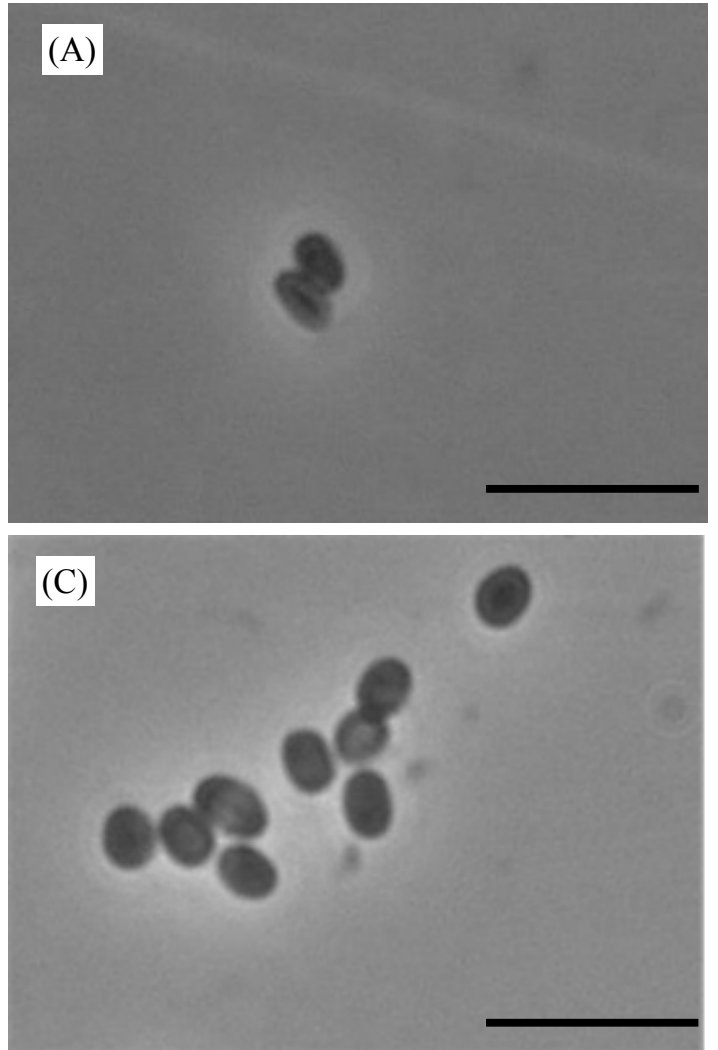

$24 \mathrm{~h}$
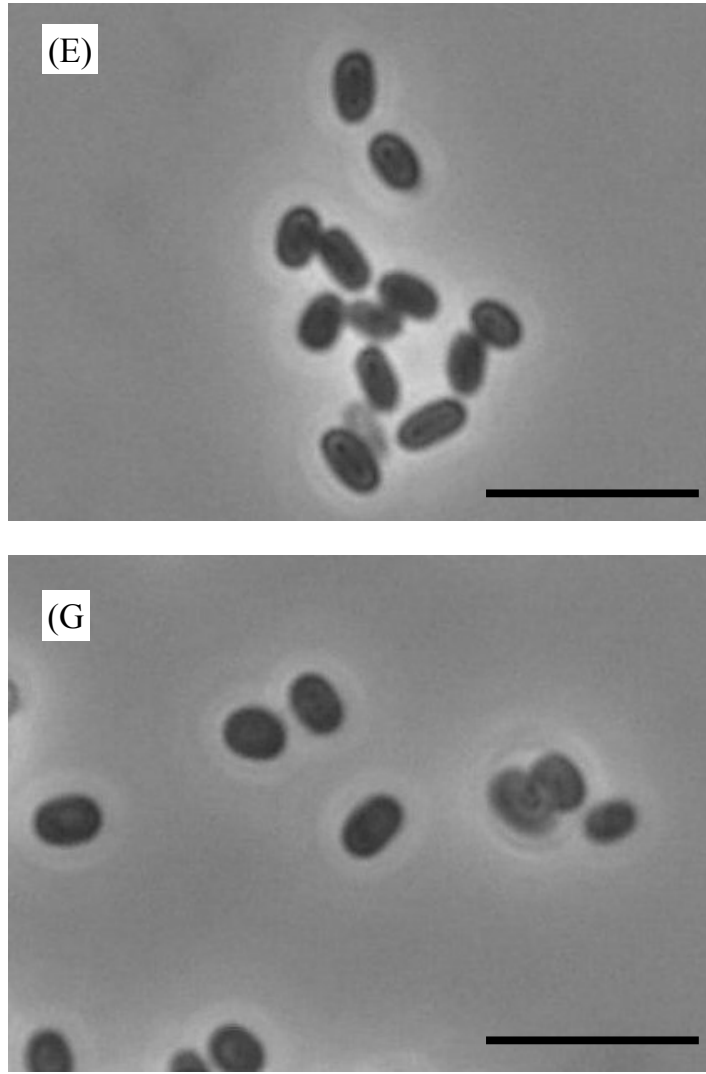

(B)

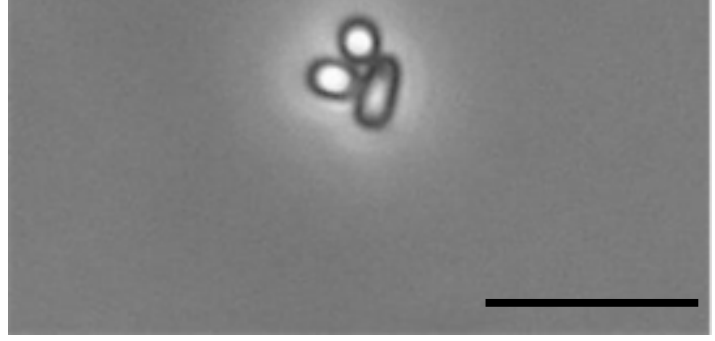

(D)

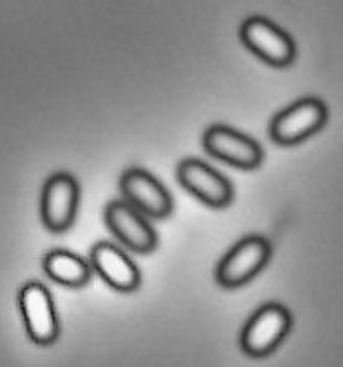

(F)

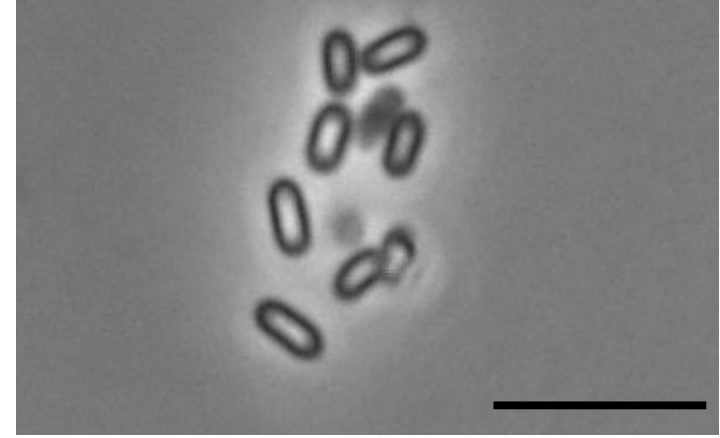

(H)
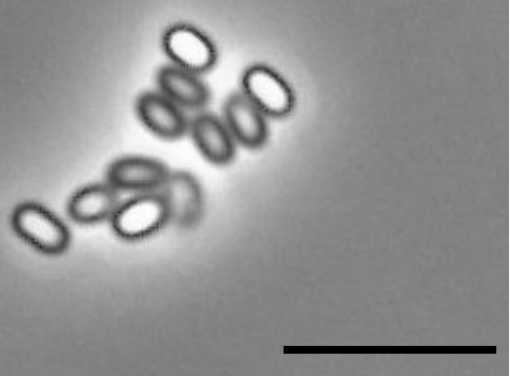

Fig. 4 

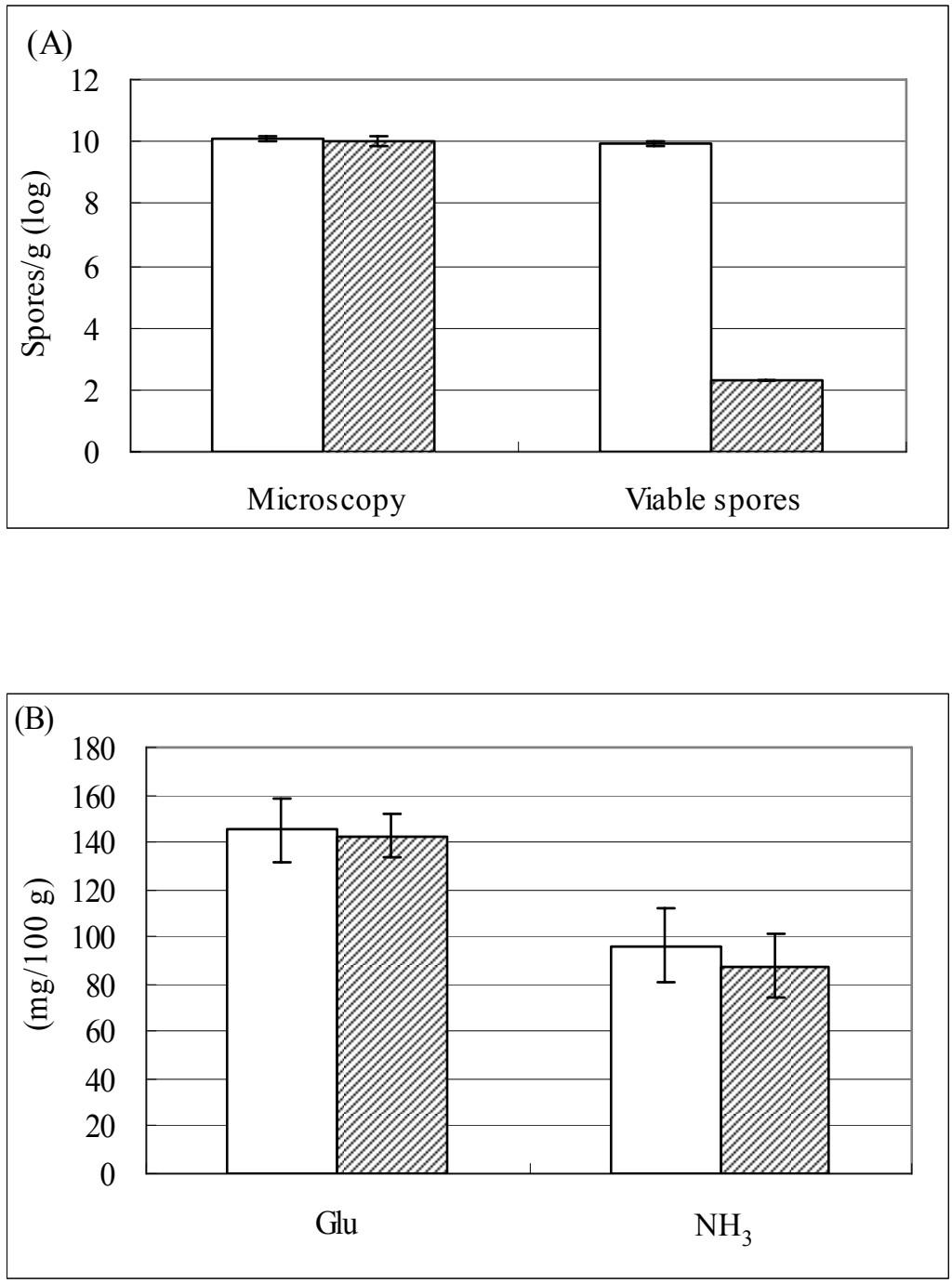

Fig. 5 